\title{
Low Resolution Rotational Spectra of Phenol
}

\author{
Ch. O., Qajar ${ }^{1}$, S. B. Kazymova ${ }^{1}$, M. R. Menzeleyev ${ }^{1} \&$ A. S. Gasanova ${ }^{1}$ \\ ${ }^{1}$ Institute of physics of National Academy of Sciences of Azerbaijan Republic, Az-1143, 33 H.Javid ave., Baku \\ Azerbaijan
}

Correspondence: S. B. Kazymova, Institute of physics of National Academy of Sciences of Azerbaijan Republic, Az-1143, 33 H.Javid ave., Baku Azerbaijan. E-mail: kazymova-s@mail.ru

Received: August 17, 2018

doi:10.5539/apr.v10n6p77
Accepted: October 20, $2018 \quad$ Online Published: November 30, 2018

URL: https://doi.org/10.5539/apr.v10n6p77

\begin{abstract}
The modeling of low-resolution (LR) microwave spectra of phenol $\mathrm{C}_{6} \mathrm{H}_{5} \mathrm{OH}$ molecule has been provided in the frequency regions of $0-700 \mathrm{GHz}$ basing on high-resolution (HR) microwave spectral data. The frequency ranges of most intensive absorption level of low-resolution microwave spectra determined. The most intensive spectral lines forming spectra extremums are developed. The recommendations related to perspectives of practical use of achieved results are given.
\end{abstract}

Keywords: rotational spectrum, high-resolution spectra, low-resolution spectra, spectrum extrema, phenol

\section{Introduction}

Changing of composition and structure of Earth atmosphere as result of human activity all over the world stipulate imminent long-term impact on average climatic indexes, lead to climatic instability, natural calamities and anomalies. Therefore, ecological monitoring of atmosphere and troposphere particularly became actual, high demanded direction of scientific researches. Thus, scientific problems and methods of detection and identification of substances dissipated in gas phased molecular compositions being, of course, very interesting for searches and investigations of molecular systems at cosmic space also taking on a great importance for local and remote atmospheric sounding purposes. Besides, methods of spectral analysis and particularly microwave spectroscopy became very perspective from the view of control on the industrial processes and logistic operations related to chemical substances with purpose of prevention of possible anthropogenic impacts on the environment as the result of accidents, leakages, failures caused by breakdowns in processes could be potentially dangerous for environment and/or personnel. Thereupon classification of some organic compounds (such as alcohols, thiols, phenols etc) as pollutants of atmosphere definitely should bring heightened interest to the investigations of their molecular composition, structure, conformational properties etc. (Zelenin, 1988; Nikitin, 2014).

Phenols - family of organic compounds classified as moderately hazardous substances and characterized by a hydroxyl $(-\mathrm{OH})$ group attached to a carbon atom that is part of an aromatic ring. Besides serving as the generic name for the entire family, the term phenol is also the specific name for its simplest member, monohydroxybenzene $(\mathrm{C} 6 \mathrm{H} 5 \mathrm{OH})$, also known as benzenol or carbolic acid. Investigation of this compound spectral characteristics were the object of series works, some of them in microwave frequency range (Kojima, 1960; Gerhards, Schmitt, \& Kleinermanns, 1996; Tanjaroon \& Kukolich, 2009; Kolesnikova, Daly, Alonso, Tercero, \& Cernicharo, 2013).

The information obtained from the results of such investigations became background data for searching, detection and identification of substances, useful both from view of environmental monitoring and cosmic space researches. It has to be noted here the atmospheric sounding methods basing on analysis of rotational and rotationally oscillatory absorption spectrums of gas-phase compounds being under pressures and temperatures relatively higher than typical for cosmic space. Besides both temperature and pressure of atmosphere are changing depends on height from sea level, weather conditions, season etc.

In line with mentioned above strict mathematical modeling of low-resolution microwave spectra of phenol $(\mathrm{C} 6 \mathrm{H} 5 \mathrm{OH})$ molecule allow to simulate different environmental condition and could be very perspective from the view of further researches in direction of design and development of devices for investigated substance detection. 
Prior to selection of technology for design of scientific tools for atmospheric sounding and searching of spectral lines of investigated substance in cosmic space, it is important to define the range of frequencies with high probability of detection, i.e. select the part of electromagnetic radiation spectra with maximum absorption. This allow to localize area of goal seeking and could be provided basing on analysis of results of theoretical modeling of low resolution spectra of investigated substances in sufficiently wide range of frequencies. In other words, modeling of low resolution spectra allow to get the data regarding presence and allocation of frequency ranges with most intensive absorption. From the view of searching and detection of substance this ranges represent the regions with most probability of success.

\section{Model of low-resolution spectra of phenol molecule}

The object of this article is development of principles and methods could apply for monitoring of atmospheric air possible containing particulate pollutants including phenol molecule and identification of presence of phenol in the interstellar space.

With this purpose the calculations of low-resolution spectra of this molecule have been provided for the frequency range 0-700Ghz. Modeling based on spectroscopic data presented in the works (Kolesnikova, Daly, Alonso, Tercero, \& Cernicharo, 2013; Tercero, Cernicharo, Pardo, \& Goicoechea, 2010; Tercero, Vincent, Cernicharo, Viti, \& Marcelino, 2011; Tercero, et al., 2012), focused also on astrophysical search for phenol molecule $(\mathrm{C} 6 \mathrm{H} 5 \mathrm{OH})$ and reported a tentative detection for this molecule in the IRAM 30m line survey of Orion KL.

The calculation model recently successfully applied for provision of researches of isopropanol and n-propanol molecules (Gasanova, 1989; Qajar Ch., Ismailzadeh, Menzeleyev, Movsumov, \& Kazymova, 2016) has been use also for the theoretical modeling of low-resolution microwave (MW) spectra of phenol. Initially, on the base of spectroscopic data represented in the work (Kolesnikova, Daly, Alonso, Tercero, \& Cernicharo, 2013) the high resolution spectra has been calculated, i.e. have been provided calculations of rotational transition frequencies and corresponding relative intensities in preliminary defined range of $0-1 \mathrm{THz}$. As the optimal appropriate limitation of total number of rotational transition taken into account have been selected transition with quantum numbers $\mathrm{J} \leq 60$ and relative intensity $\geq 10 \mathrm{e}^{-6} \mathrm{~cm}^{-1}$. Calculations have been provided for ground $v_{0}$ and also excited $v_{\mathrm{b}}, v_{\mathrm{t}}$ states. The calculation results show significant decreasing of spectral lines intensities after $700 \mathrm{GHz}$ so further modeling of low-resolution MW spectra provided in the $0-700 \mathrm{GHz}$ frequency range (fig.1).

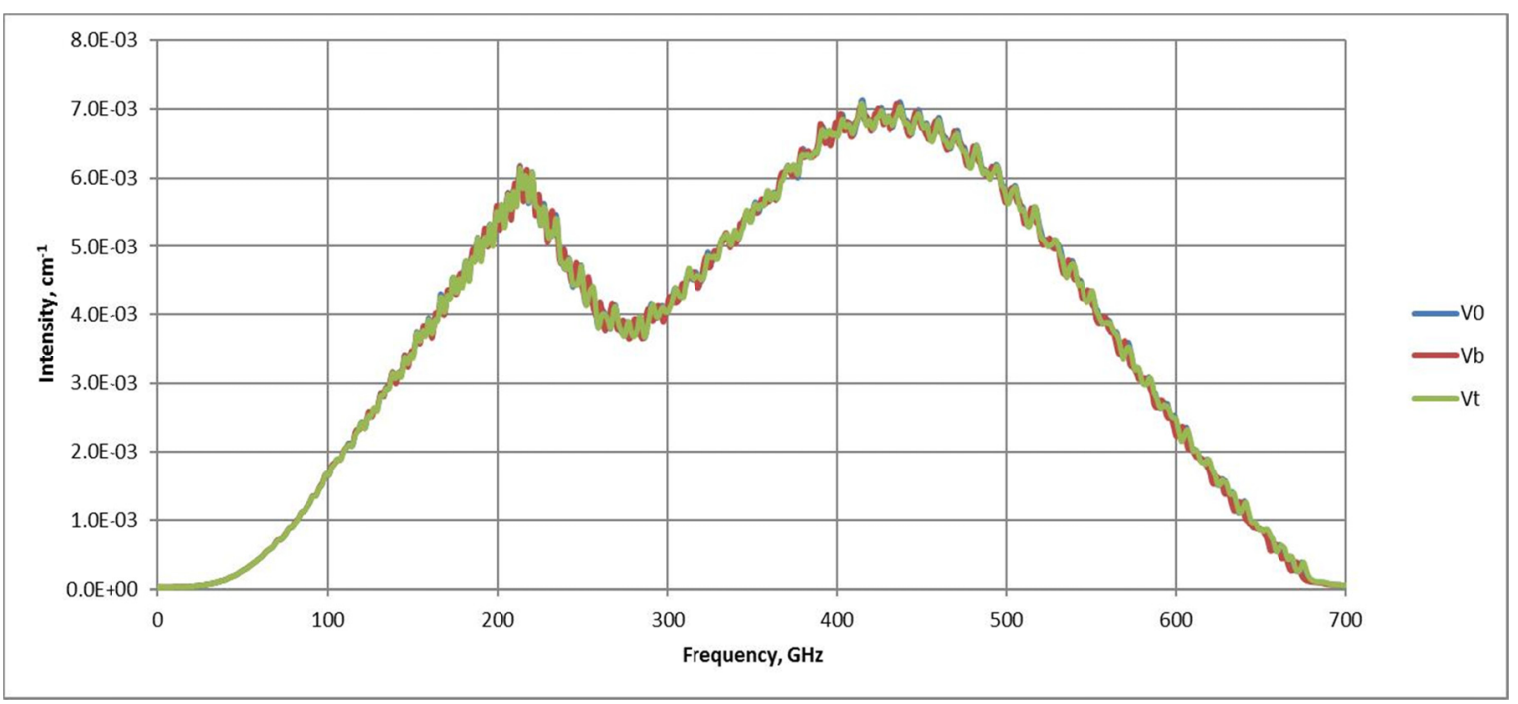

Fig.1 Low-resolution spectra of phenol in the $0-700 \mathrm{GHz}$ frequency range

The value of spectral lines half width has been conditionally selected as $2 \mathrm{GHz}$ to get the Lorentz profile shape enough smooth to allow confident identification of extremums of the of low-resolution spectra of investigated molecule.

The spectrums of ground and excited states on spectrogram on fig.1 looks almost identical, therefore further modeling of low-resolution spectra additionally provided also for each of absorption maximums frequency 
ranges located at $190-240 \mathrm{GHz}$ and $380-450 \mathrm{GHz}$ accordingly. This time the value of half width of spectral lines were selected as $1 \mathrm{GHz}$ to let us examine both ranges with "increased resolution" (fig.2 \& 3).

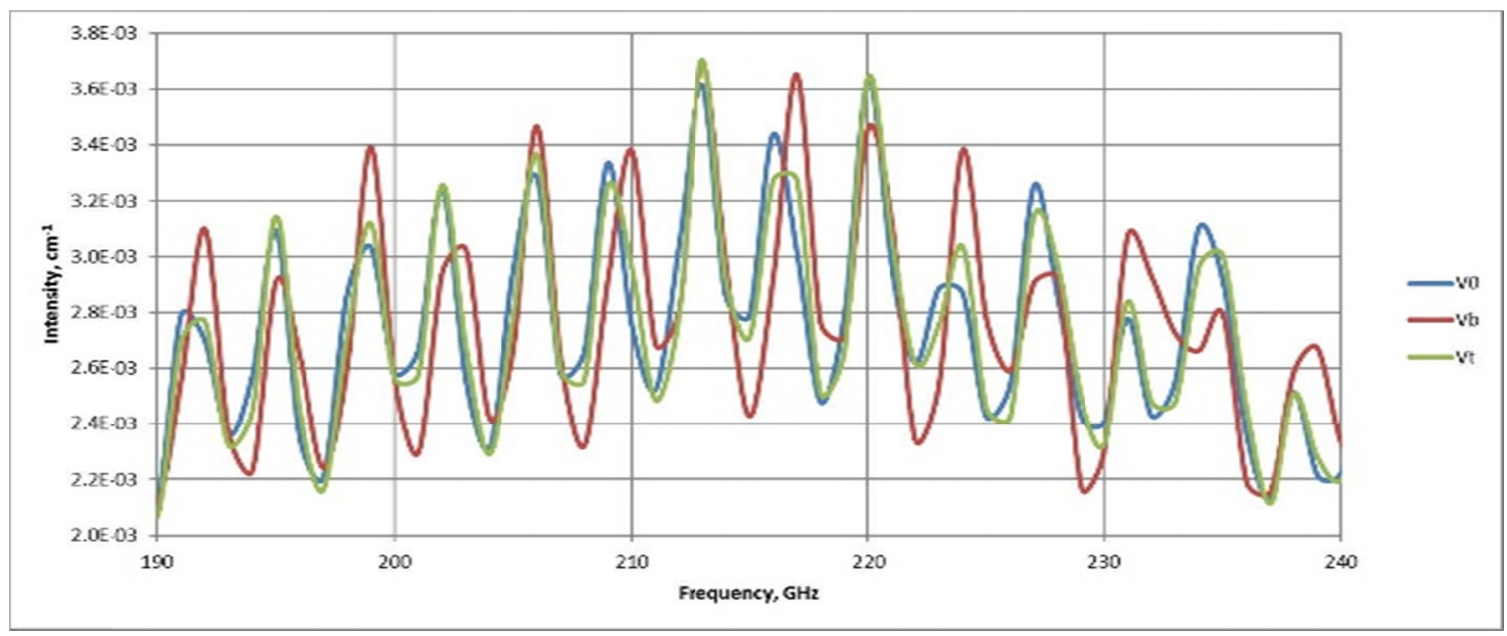

Fig.2 Low-resolution spectra of phenol in 190-240GHz frequency range

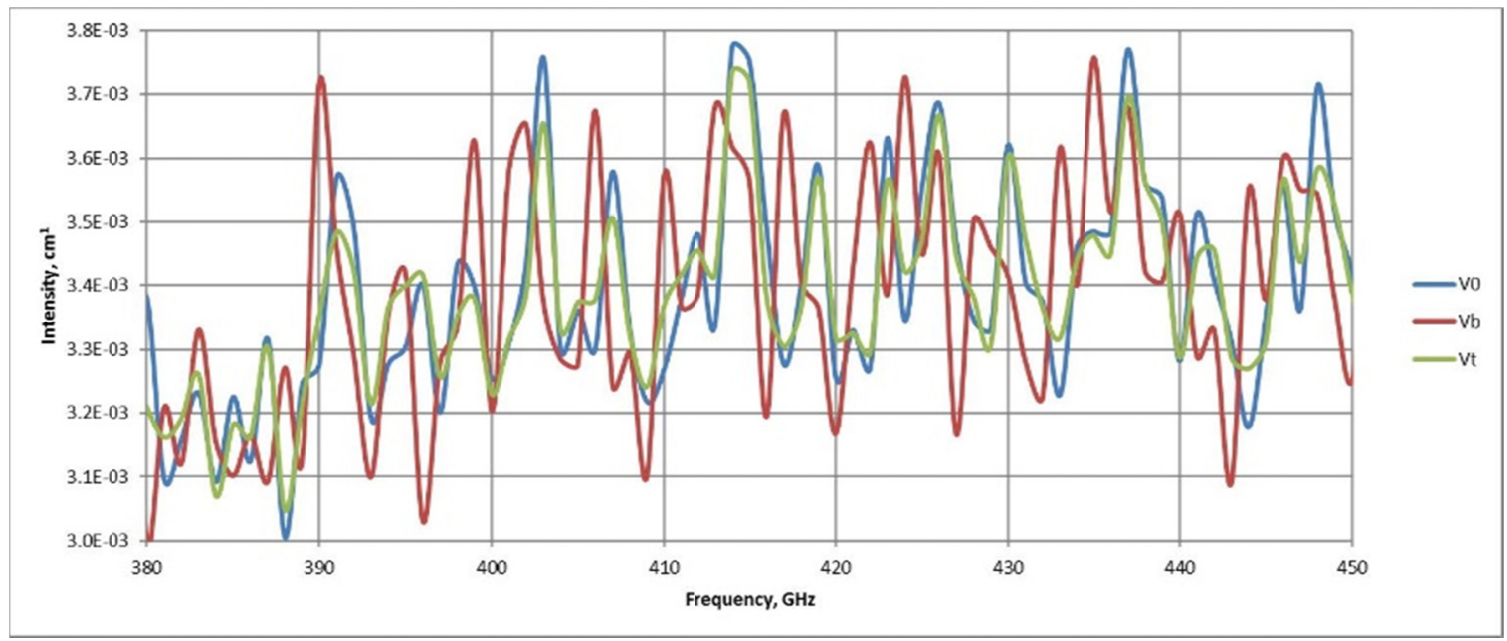

Fig.3 Low-resolution spectra of phenol in $380-450 \mathrm{GHz}$ frequency range

As it stated above, the purpose of this work is development of theoretical model of low-resolution spectra of $\mathrm{C} 6 \mathrm{H} 5 \mathrm{OH}$ phenol molecule and localization of frequency ranges with maximal intensity of absorption spectra in microwave region. However in case of researches related with searching and detection of molecules in conditions typical for interstellar space, i.e. for pressures near to absolute vacuum and extremely low temperatures special interest may be aroused not only in frequency range most probable for detection, but also in most intensive lines directly forming absorption extremums. For this purpose the modeling of high-resolution spectra of ground state of phenol has been provided for narrow frequency ranges matching to the absorption maximums of previously examined low-resolution spectra ranges (fig $4 \& 5$ ).

Analysis of spectrograms represented on fig. $4 \& 5$ allows to state presence of series of lines with high relative intensity in frequency ranges $210-215 \mathrm{GHz}$ and $410-420 \mathrm{GHz}$. For the first range extremum formed by the lines related to quantum transitions with $\mathrm{J}=56-59$, for second one - to quantum transitions with $\mathrm{J}=37-42$.

Thus, the findings of provided modeling could be useful both for identification of presence of certain molecular system in gas phased molecular compositions being under high pressures and temperatures conditions and for selection of frequency ranges most perspective for detection of spectral lines of defined substance during provisions of astronomical researches of interstellar space. 


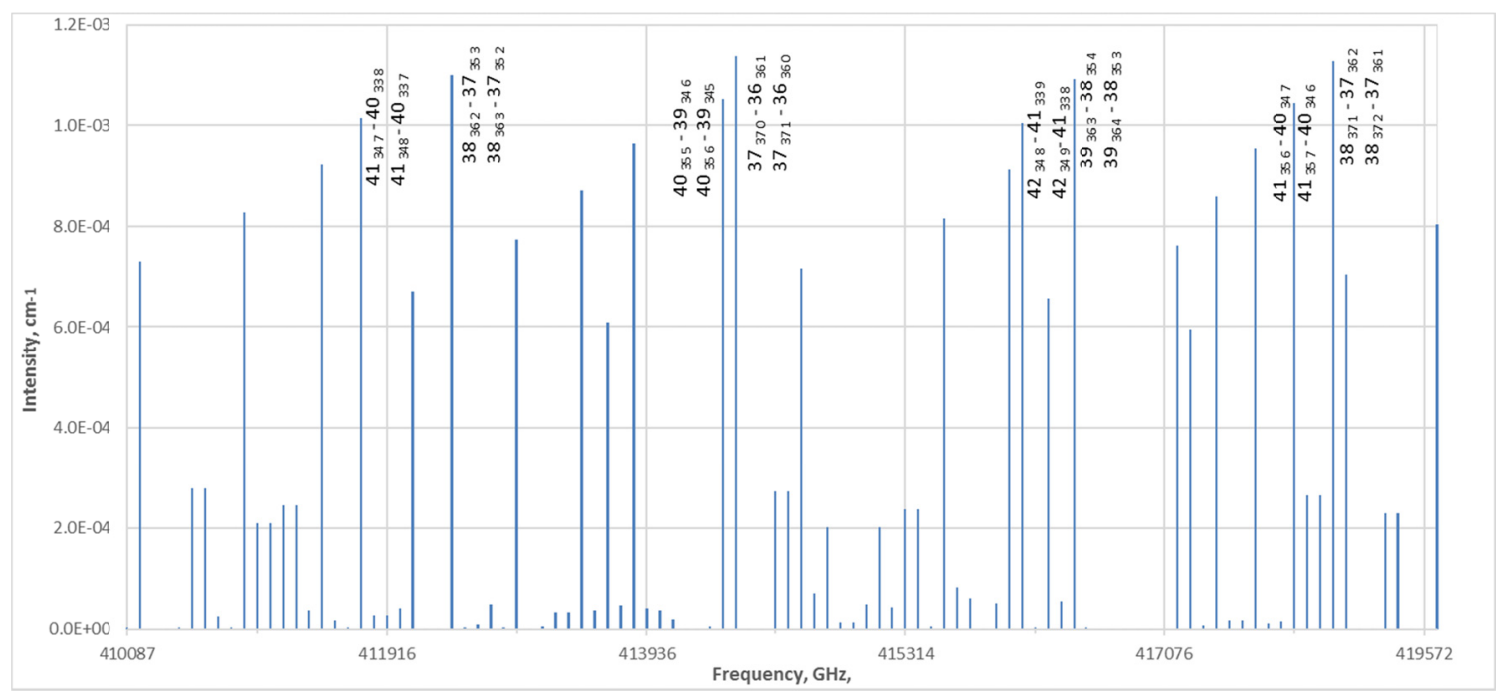

Fig.4 High-resolution spectra of phenol in $210-215 \mathrm{GHz}$ frequency range

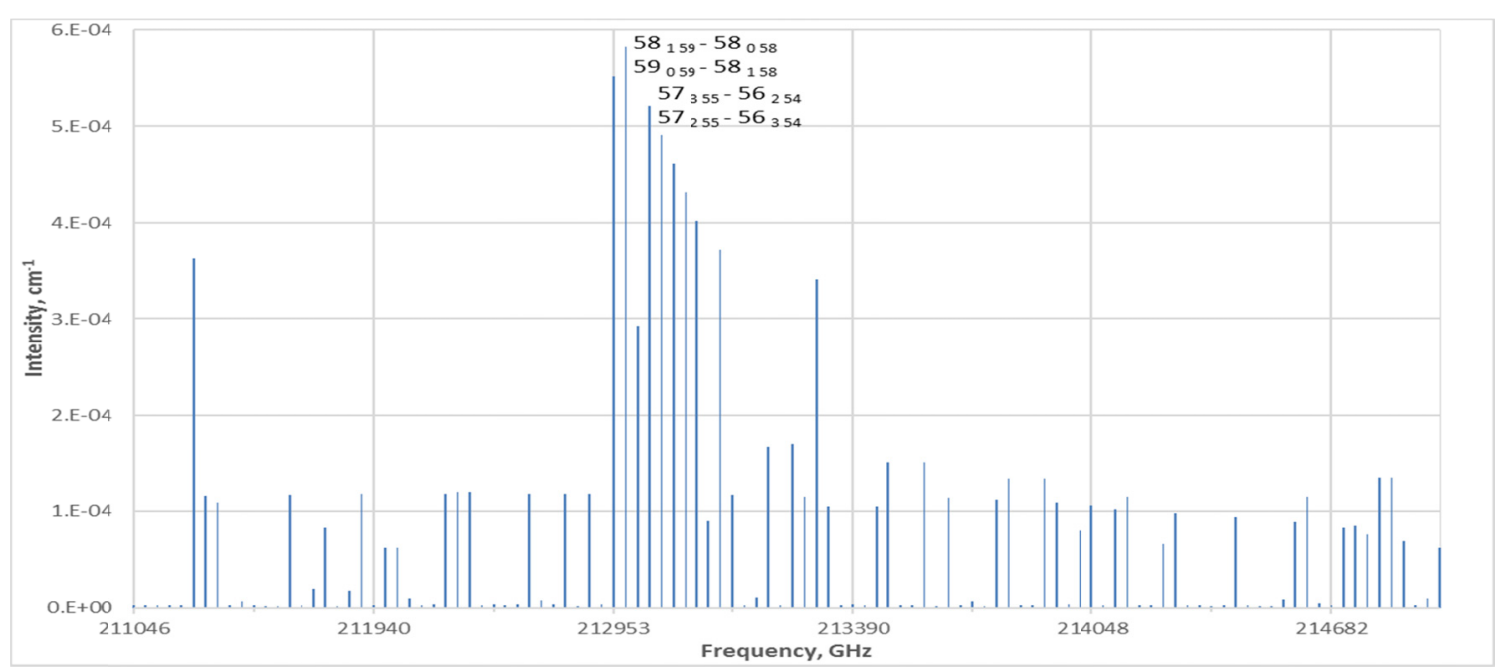

Fig.5 High-resolution spectra of phenol in $410-420 \mathrm{GHz}$ frequency range

\section{Discussion}

Investigations of low-resolution spectrums of gas-phased molecules let collect the data regarding values of frequencies relevant to maximums of absorption spectra of defined molecular systems. This could be very interesting and important from the view of environment ecological problems solving, researches of practical and fundamental tasks of atmospheric physics, especially in the question of definite assignment of emission spectrums of gas-phased atmospheric inclusions to certain investigated substance.

The results obtained in this job allow recommending searching and identification of phenol molecule in two frequency ranges $190-240 \mathrm{GHz}$ and $380-450 \mathrm{GHz}$ accordingly. Besides, the first range could be advised for sounding in atmospheric conditions, as it has clearly defined extremum of low-resolution spectral curve and relatively higher values of quantum numbers of the spectral lines forming this extremum. The second range contains transitions that are more intensive and allocated relatively wider within the range, so it more sutable for astronomic researches. These also comply with specific of scientific tools using in both cases: higher operational frequency of devices is typical for equipment designed for special purposes (such as for astronomical researches), initially expensive and require for high-qualified personnel. Relatively low frequency and clearly identified extremum allow using more cost effective, simple in use devices. 


\section{Acknowledgement}

This work was supported by the Science Development Foundation under the President of the Republic of Azerbaijan - Grant № EİF-BGM-3-BRFTF-2 ${ }^{+} / 2017-15 / 03 / 1$

\section{References}

Gasanova, A. S. (1989). Millimeter and submillimeter range rotational spectra (high J), double MW-RF resonances and structure of trans-conformer of isopropanol molecule. Dissertation for a degree of philosophy doctor in physics and mathematics, Baku 175.

Gerhards, M., Schmitt, M., \& Kleinermanns, K.. (1996). The structure of phenol $\left(\mathrm{H}_{2} \mathrm{O}\right)$ obtained by microwave spectroscopy. The Journal of Chemical Physics, 104, 967

Kojima, T. (1960). Potential Barrier of Phenol from its Microwave Spectrum. J. Phys. Soc. Japan., 15, 284-287

Kolesnikova, L., Daly, A. M., Alonso, J. L., Tercero, B., \& Cernicharo, J. (2013). The millimeter wave tunneling-rotational spectrum of phenol. Journal of Molecular Spectroscopy, 289, 13-20.

Qajar Ch., O., Ismailzadeh, G. I., Menzeleyev, M. R., Movsumov, I. Z., \& Kazymova, S. B. (2016). On the simulation of low-resolution microwave spectra of n-propanol in the gas phase. Journal of applied spectroscopy, 83(3), 385-389.

Nikitin, O. V. (2014). Control of sources of atmospheric air pollution. Kazan, University of Kazan.

Tanjaroon, C., \& Kukolich, S. G. (2009). Measurements of the rotational spectra of phenol and 2-pyrone and computational studies of the H-bonded phenol-pyrone dimer. J Phys.Chem. A., 113, 32,

Tercero, B., Cernicharo, J., Pardo, J. R., \& Goicoechea, J. R. (2010). A line confusion limited millimeter survey of Orion KL I. Sulfur carbon chains-I. Sulfur carbon chains. Astronomy \& Astrophysics, 517, A96.

Tercero, B., Margulès, L., Carvajal, M., Motiyenko, R. A., Huet, T. R., Alekseev, E. A., ... \& Cernicharo, J. (2012). Microwave and submillimeter spectroscopy and first ISM detection of 18O-methyl formate. Astronomy \& Astrophysics, 538, A119.

Tercero, B., Vincent, L., Cernicharo, J., Viti, S., \& Marcelino, N. (2011). A line-confusion limited millimeter survey of Orion KL-II. Silicon-bearing species. Astronomy \& Astrophysics, 528, A26.

Zelenin, K. N. (1988). Organic substances of atmosphere. ISSEP Soros educational journal, 4, 39-44

\section{Copyrights}

Copyright for this article is retained by the author(s), with first publication rights granted to the journal.

This is an open-access article distributed under the terms and conditions of the Creative Commons Attribution license (http://creativecommons.org/licenses/by/4.0/). 